\title{
Characterisation of the Fast-Ion Edge Resonant Transport Layer Induced by 3D Perturbative Fields in the ASDEX Upgrade Tokamak through Full Orbit Simulations
}

L. Sanchis ${ }^{1,2}$, M. Garcia-Munoz ${ }^{1,2}$, A. Snicker ${ }^{3}$, D. A. Ryan ${ }^{4}$, D. Zarzoso $^{5}$, L. Chen ${ }^{6}$, J. Galdon-Quiroga ${ }^{1,2}$, M. Nocente ${ }^{7}$, J. F. Rivero-Rodriguez $^{1,2}$, M. Rodriguez-Ramos ${ }^{2}$, W. Suttrop ${ }^{8}$, M. A. Van Zeeland ${ }^{9}$, E. Viezzer ${ }^{1,2}$, M. Willensdorfer ${ }^{8}$, F. Zonca ${ }^{10}$ the ASDEX Upgrade Team and the EUROfusion MST1 Team

1 Dept. of Atomic, Molecular and Nuclear Physics, University of Seville, Avda.

Reina Mercedes, 41012 Seville, Spain

2 Centro Nacional de Aceleradores CNA (Universidad de Sevilla, Junta de

Andalucía, CSIC), Avda. Thomas A. Edison 7, 41092 Seville, Spain

${ }^{3}$ Dept. of Applied Physics, Aalto University, FI- 00076, Aalto, Finland

${ }^{4}$ CCFE, Culham Science Centre, OX14 3DB, Abingdon, UK

${ }^{5}$ Aix-Marseille Université, CNRS, PIIM, UMR 7345, Mareseille, France

${ }^{6}$ IFTS, Zhejiang University, 310027, 310027, Hangzhou, China

7 Universita degli Studi di Milano-Bicocca, Piazza della Scienza 3, 20126, Milano, Italy

${ }^{8}$ Max Planck Institute for Plasma Physics, Boltzmannstr. 2, 85748 Garching, Germany

${ }^{9}$ General Atomics, CA 92186-5608, San Diego, USA

10 ENEA C.R. Frascati, CP 65-00044, Frascati, Italy

E-mail: Isanchis@us.es

\begin{abstract}
In recent experiments at the ASDEX Upgrade tokamak the existence of an Edge Resonant Transport Layer (ERTL) was revealed as the main transport mechanism responsible for the measured fast-ion losses in the presence of externally applied 3D fields. The Monte Carlo orbit-following code ASCOT was used to study the fast-ion transport including the plasma response calculated with MARS-F, reproducing a strong correlation of fast-ion losses with the poloidal mode spectra of the 3D fields. In this work, a description of the physics underlying the ERTL is presented by means of numerical simulations together with an analytical model and experimental measurements to validate the results. The degradation of fast-ion confinement is calculated in terms of the variation of the toroidal canonical momentum $\left(\delta P_{\phi}\right)$. This analysis reveals resonant patterns at the plasma edge activated by $3 \mathrm{D}$ perturbations and emphasizes the relevance of nonlinear resonances. The impact of collisions and the radial electric field on the ERTL is analyzed.
\end{abstract}

$\ddagger$ See the author list of Overview of progress in European Medium Sized Tokamaks towards an integrated plasma-edge/wall solution by H. Meyer et al., Nucl. Fusion 571020142017. 


\section{Introduction}

Edge Localized Modes (ELMs), present in high confinement regimes in current tokamak plasmas, must be kept under control to limit energy and particle losses, which at current levels would be unacceptable in future fusion devices such as ITER. Therefore, substantial efforts are being focused on the development of reliable techniques capable of suppressing or mitigating these instabilities in high confinement regimes. One of the leading techniques among them is the application of external 3D magnetic perturbations (MPs) [1, 2, 3, 4]. These perturbative fields have, however, an impact on the overall plasma stability and confinement. The plasma response depends on several factors such as magnetic equilibrium, main plasma parameters and the applied perturbation spectrum. The mechanism responsible for the ELM mitigation has been found to depend strongly on the plasma response to the applied MP $[5,6]$ and its poloidal mode spectra [7, 8]. Considering this, the effect of the poloidal mode spectrum from the external MPs and of the plasma response generated by the perturbation, needs to be analysed. This effect is most pronounced on the energetic particles with relatively long slowing-down times [9] and must be carefully assessed, as they will be present in future fusion devices. Previous numerical simulations of the impact on ELM control coils on fast-ion confinement in ITER $[10,11]$ have predicted up to a $20 \%$ power loss for the neutral beam injection (NBI) and $6 \%$ for fusion born alpha particles. First experiments at ASDEX Upgrade (AUG) and DIII-D have shown that the externally applied perturbations can lead to a degradation of the energetic particle confinement $[12,13,14]$ for certain magnetic configurations, compromising the integrity of the device. Experiments on both AUG and DIII-D show that the poloidal mode spectra of the applied 3D resonant magnetic perturbation commonly used to mitigate ELMs can greatly affect the fast-ion confinement [15]. In this paper, we report on the existence of an Edge Resonant Transport Layer (ERTL) responsible for the fast-ion losses observed in the presence of externally applied 3D fields. Full orbit simulations carried out with the ASCOT [16] code using the plasma response calculated with MARS-F [17] reproduce a strong correlation of the fast-ion losses with the 3D fields poloidal mode spectra. This work presents an analysis of fast-ion confinement in terms of the variation of the toroidal canonical momentum $\left(\delta P_{\phi}\right)$, which reveals resonant structures at the plasma edge activated by $3 \mathrm{D}$ fields that match rational orbital resonances.

This paper is structured as follows: Section 2 describes the experiments carried out at AUG. In section 3, the modelling techniques using the full-orbit code ASCOT and MARS-F, which is a single fluid, full MHD eigenvalue code in full toroidal geometry, are discussed. Section 4 introduces the ERTL and describes the resonant transport mechanism that arises due to the $3 \mathrm{D}$ fields. In section 5 , the impact of the findings presented with respect to fast-ion transport is assessed. A summary and conclusions are given in section 6 . 


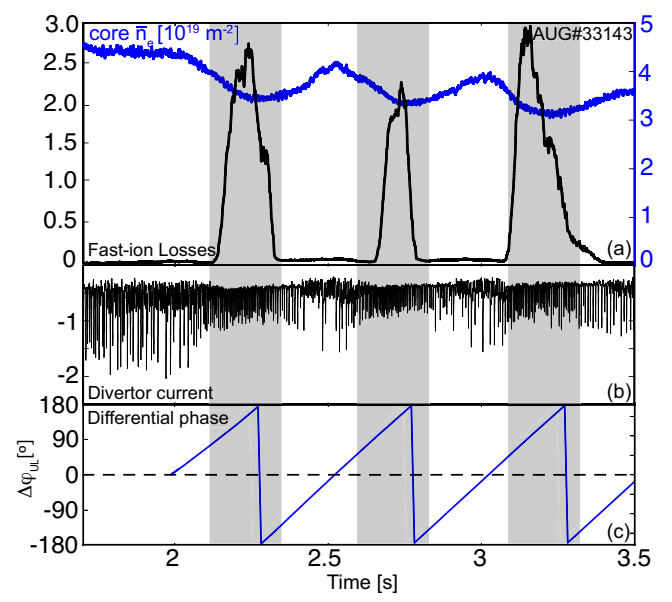

Figure 1. AUG\#33143. (a) Normalised FILD signal (black) and plasma core line-integrated electron density (blue) as functions of time. (b) Poloidal thermocurrents from the outer divertor target plates showing ELM mitigation as a function of time and (c) time window corresponding to the applied current phase $\Delta \varphi_{U L}$.

\section{Experiment}

Several experiments have been carried out in AUG to show the impact of MPs poloidal mode spectra on ELM mitigation, in particular, shot \#33143 with high normalised $\beta_{N}=2.4$, low collisionality $\nu_{e}^{*}=0.2$ and $q_{95}=3.8$. A magnetic perturbation with a toroidal mode number $n=2$ was generated by the ELM control coils installed in AUG, where currents flowing through the lower set of coils were kept constant in time while the upper currents were slowly rotating at $2 \mathrm{~Hz}$, introducing a phase shift $\left(\Delta \varphi_{U L}=\phi_{U}-\phi_{L}\right)$ that resulted in a continuous scan in the poloidal mode spectra [18]. The source of energetic particles was introduced by means of $5 \mathrm{MW}$ neutral beam injection (NBI) distributed on 2 sources with $2.5 \mathrm{MW}$ from $2 \mathrm{NBI}$ boxes, one at $60 \mathrm{kV}$ (NBI\#3) the other at $93 \mathrm{kV}$ (NBI\#8). Figure 1 shows density pump out and partial ELM mitigation when the coils are turned on, but also a modulation with the poloidal mode spectra. The effect of this modulation is especially clear in the Fast Ion Lost Detector (FILD) [19] measurements, where fast-ion losses are only detected with certain coil configurations (figure 1) between $\Delta \varphi_{U L}=100^{\circ}$ and $\Delta \varphi_{U L}=220^{\circ}$.

\section{Modelling}

Full orbit simulations of the observed fast-ion transport induced by the externally applied 3D fields have been carried out using the ASCOT code, a realistic fast-ion distribution and the 3D perturbations fields calculated using the vacuum approach and the plasma response given by MARS-F [20]. The magnetic fields were generated as the combination of the axisymmetric equilibrium provided by the CLISTE code [21] with the 3D perturbation calculated by MARS-F and interpolated in ASCOT from a cylindrical 
Characterisation of the ERTL Induced by 3D Fields in AUG through FO Simulations 4
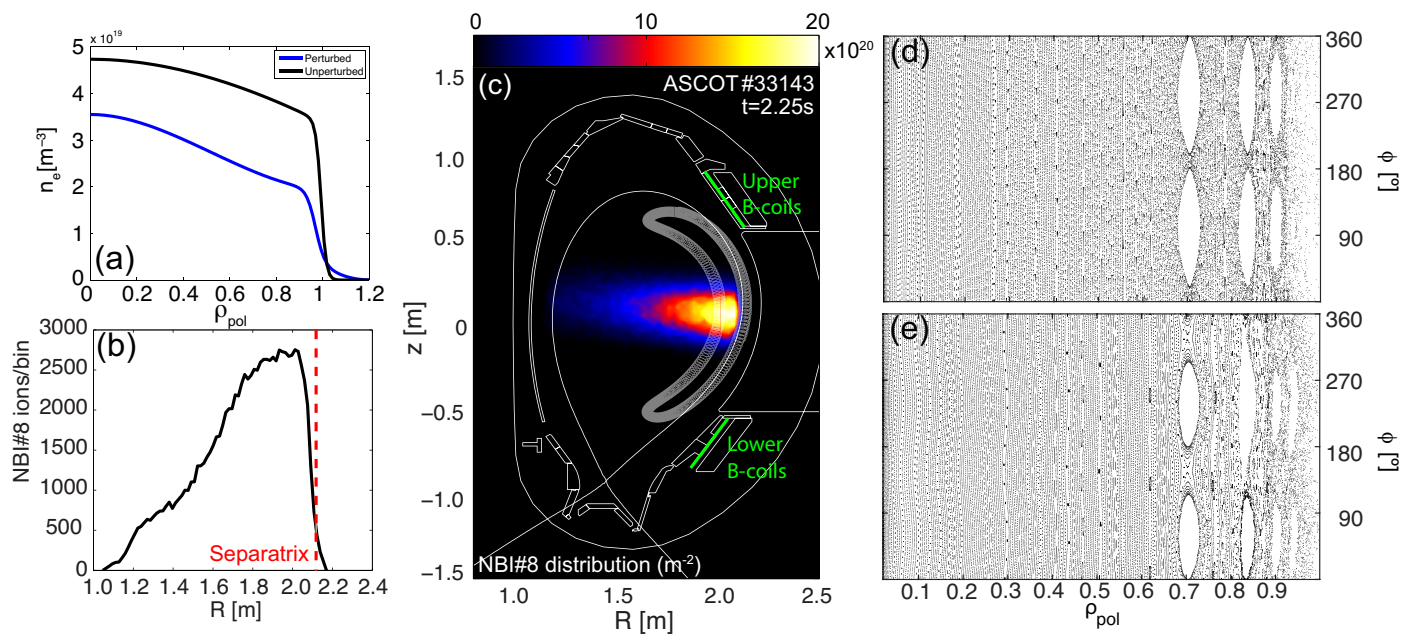

Figure 2. AUG\#33143. (a) Electron density profiles showing the unperturbed profile (black) measured at $I_{\text {coil }}=0(t=1.80 \mathrm{~s})$ and the profile during density pump-out produced by the MP coils at $t=2.25 \mathrm{~s}$ (blue). (b) NBI birth profile distribution as a function of the plasma major radius. (c) Poloidal cross section of AUG vessel including the NBI\#8 deposition using the perturbed density profile and a representative trapped orbit. Toroidal Poincaré plots showing the $n=2 M P$ perturbation for: (d) $\Delta \varphi_{U L}=40^{\circ}$, (e) $\Delta \varphi_{U L}=260^{\circ}$

grid using splines. The viability of this model is based on its capability of reproducing the measured data. To that end shot \#33143 was used to validate the simulations. In this case, the initial fast-ion distribution was calculated for NBI\#8 as this beam is more energetic and presents more associated fast-ion losses. The beam deposition was calculated from the profiles at the phase where coils are turned on (figure 2(a)) as the ion deposition in the scrape-off layer (figure $2(\mathrm{~b})$ and (c)) is very sensitive to density variations, entailing a significant impact on fast-ion losses. As will be discussed later, more information about the NBI\#8 pitch distribution is shown in figure 4.

The 3D perturbation fields are calculated at several time points spanning the entire spectra. Figures $2(\mathrm{~d})$ and (e) present the toroidal Poincaré plots corresponding to the configurations $\Delta \varphi_{U L}=40^{\circ}$ and $\Delta \varphi_{U L}=260^{\circ}$ calculated with MARS-F including the rotational shielding of the MP. These plots show the magnetic island structures appearing at the region $\rho_{\text {pol }}=0.7-1.0$ caused by the applied perturbation.

The fast-ion transport induced by each perturbation fields, i.e. time point or coils configuration, has been calculated using orbit simulations including the gyromotion. For this purpose, the ASCOT leap-frog method with a time step of $10^{-9} \mathrm{~s}$ has been used. The perturbation fields include the main harmonic perturbation $n=2$ as well as the secondary harmonic $n=6$ given by the realistic geometry of the MP coils. Figure 3(a) shows the total losses (normalised by their maximum) obtained with ASCOT for $5 \mathrm{~ms}$ simulation time for each MP configuration using the same initial particle distribution. All cases show a similar trend with the coils configuration, i.e. poloidal mode spectrum, with the minimum losses obtained when a pure $n=2$ perturbation is applied using the 
Characterisation of the ERTL Induced by 3D Fields in AUG through FO Simulations 5

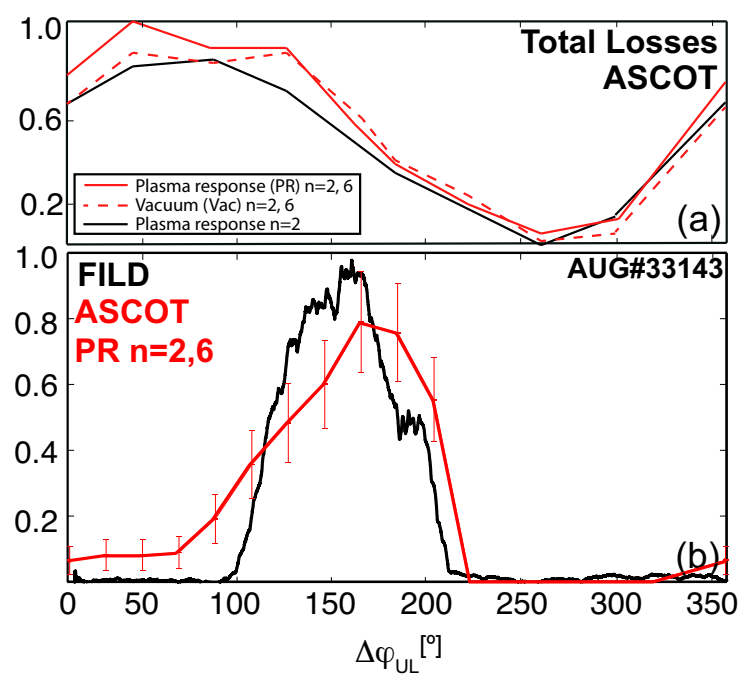

Figure 3. (a) AUG\#33143. (a) Total fast-ions losses normalised to its maximum value as a function of $\Delta \varphi_{U L}$ including the $n=2$ plasma response (black), the $n=2,6$ plasma response (red) and the $n=2,6$ vacuum fields without the plasma response (red dashed line). (b) Normalised values of measured (black) and simulated with ASCOT (red) FILD signals as a function of the applied $\Delta \varphi_{U L}$.

vacuum approach. These results also reveal that the impact of the plasma response on energetic particle losses changes with the MP poloidal mode spectra. While the plasma response and vacuum approach for $n=2,6$ lead to similar fast-ion losses at $\Delta \varphi_{U L}=260^{\circ}$, the plasma response can increase the losses up to $20 \%$ at $\Delta \varphi_{U L}=20^{\circ}$. Excluding prompt losses, i.e. particles lost to the walls during the first poloidal bounce, most of the losses induced by the 3D fields occur within the first $5 \mathrm{~ms}$, so we can assume that the chosen following time is representative of the fast-ion loss behaviour.

Figure 3(b) shows the fast-ion losses measured with the FILD detector during these experiments. The FILD system used in these experiments is located above the midplane at $\mathrm{z}=0.30 \mathrm{~m}, \mathrm{R}=2.19 \mathrm{~m}$ and $\phi=123.5^{\circ}$. The detector geometry allows the detection of particles within a pitch range between $\Lambda=-0.9$ and $\Lambda=-0.1$ and gyroradius between $\rho_{L}=20 \mathrm{~mm}$ and $\rho_{L}=120 \mathrm{~mm}$. FILD measures fast-ion losses when the perturbation fields have a $\Delta \varphi_{U L}$ between $100^{\circ}$ and $200^{\circ}$ with a maximum at $\Delta \varphi_{U L} \approx 150^{\circ}$. A reasonable good agreement, see red curve in Figure 3(b), is obtained with ASCOT when filtering the lost particles that hit the FILD head, which is included as a 3D element of the AUG wall. Charge-exchange losses were neglected in this numerical model and assumed not to play a key role in the understanding of the fast-ion transport dependency on the 3D fields configuration. 


\section{Edge Resonant Transport Layer (ERTL)}

The experimental results show that $\Delta \varphi_{U L}$ has a strong effect on fast-ion losses. Using the model described in the previous section, the phase-space of fast-ions affected by the 3D perturbation was calculated through the variation of the toroidal canonical momentum $\left(P_{\phi}\right)$ as a function of $\Delta \varphi_{U L} . P_{\phi}$ of a charged particle is defined as:

$$
P_{\phi}=m R v_{\phi}-Z e \psi
$$

where $m$ is the particle mass, $R$ is the major radius, $v_{\phi}$ is the toroidal component of the velocity, $Z e$ is the particle charge and $\Psi$ is the poloidal flux. $P_{\phi}$ is a constant of motion for axisymmetric fields, but with the inclusion of non axisymmetric magnetic fields leading to a toroidal symmetry breaking, $P_{\phi}$ is not an invariant and its variation is associated to a radial transport of the particles. In this context, a positive variation in $P_{\phi}$ means that the particle is drifting inwards, while a negative variation leads the ion to drift outwards. The variation of the fast-ion $P_{\phi}\left(\left\langle\delta P_{\phi}\right\rangle\right)$ was calculated as the time averaged variation of $P_{\phi}$ along each particle trajectory as follows:

$$
\left\langle\delta P_{\phi}\right\rangle=\frac{\sum_{i=1}^{N} P_{\phi}(i)-P_{\phi}(0)}{N},
$$

where $P_{\phi}(0)$ is the initial value of $P_{\phi}$ at $t=0, i$ indicates different time points along the particle orbit and $N$ is the total number of time points used for the average. $\left\langle\delta P_{\phi}\right\rangle$ is shown in figure 4 as a function of the particle pitch $\Lambda=v_{\|} / v_{t o t}$ and the plasma major radius. Clear patterns appear for passing $(\Lambda<-0.5$ at $\mathrm{R}=2.0 \mathrm{~m})$ and trapped $(\Lambda>-0.5$ at $\mathrm{R}=2.0 \mathrm{~m}$ ) ions with a maximal $\left\langle\delta P_{\phi}\right\rangle$ located within $10 \mathrm{~cm}$ around the separatrix. The transport of trapped ions is dominated by resonant structures for all particle radial locations, while passing ions also show non-structured patterns from $\mathrm{R}=2.10 \mathrm{~m}$. These structures also show a strong dependency with the particle initial pitch angle. $\left\langle\delta P_{\phi}\right\rangle$ is shown in figures 5(a)-(c) as a function of the particle energy and initial major radius with particle pitch $\Lambda=-0.5$ set by the NBI\#8 injection geometry and initial $z$ on the midplane, where $\left\langle\delta P_{\phi}\right\rangle$ was calculated through full orbit simulations for different coil configurations to show the impact of $\Delta \varphi_{U L}$ on energetic particle transport.

In figures 5 (a)-(c), clear transport structures are observed within $5 \mathrm{~cm}$ around the separatrix. The maximum in simulated total losses presented in figure 3 (a) for $\Delta \varphi_{U L}=40^{\circ}$ appears correlated with a maximum outward transport as figure 5 (a) shows while the minimum in simulated total losses for $\Delta \varphi_{U L}=260^{\circ}$ appears with a maximum inward transport as figure 5 (c) shows. An intermediate configuration for $\Delta \varphi_{U L}=160^{\circ}$ leads to intermediate total losses that are reflected in a moderate transport $\left(\left\langle\delta P_{\phi}\right\rangle\right)$ as figure $5(\mathrm{~b})$ shows.

The patterns observed in the $\left\langle\delta P_{\phi}\right\rangle$ plots can be understood by comparing them to the particle resonances. We use the static equilibrium for discharge AUG\#33143 to calculate the particle resonances as the ratio between the bounce $\left(\omega_{b}\right)$ and precessional frequency $\left(\bar{\omega}_{d}\right)$ presented in figs $5(\mathrm{a})-(\mathrm{c})$. In figures $6(\mathrm{a})$ and (b) the frequency ratio has 
Characterisation of the ERTL Induced by 3D Fields in AUG through FO Simulations 7

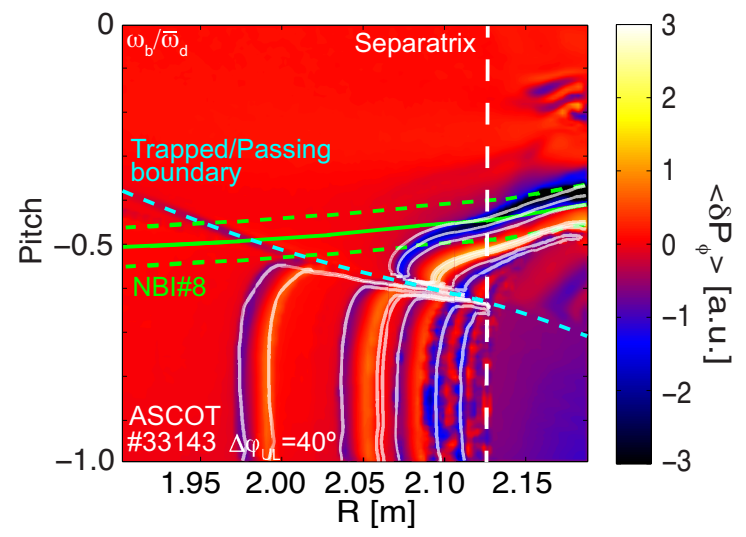

Figure 4. AUG\#33143. $\left\langle\delta P_{\phi}\right\rangle$ as a function of plasma major radius and particle pitch angle for a particle with energy $E=60 \mathrm{keV}$ and started at $z=0$. The fast-ion NBI\#8 distribution maximum (green) and FWHM (dashed green). Blue curve indicates the location of the trapped/passing boundary and white contours show the particle frequency ratio $\omega_{b} / \bar{\omega}_{d}$
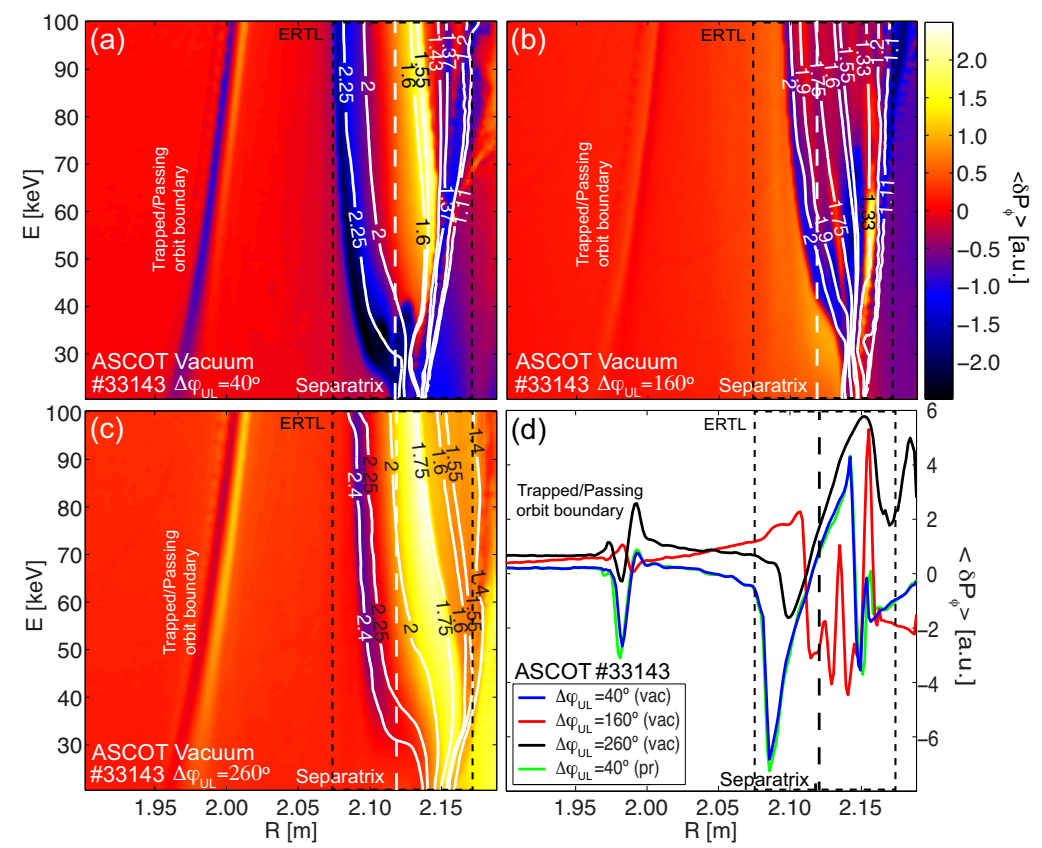

Figure 5. $A U G \# 33143 .\left\langle\delta P_{\phi}\right\rangle$ as a function of particle major radius and energy for: (a) $\Delta \varphi_{U L}=40^{\circ}$, (b) $\Delta \varphi_{U L}=160^{\circ}$, (c) $\Delta \varphi_{U L}=260^{\circ}$. White contours indicate the particle frequency ratio $\left(\omega_{b} / \bar{\omega}_{d}\right)$. (d) Radial profiles of $\left\langle\delta P_{\phi}\right\rangle$ for different $\Delta \varphi_{U L}$ at energy of $E=50 \mathrm{ke} V$ and pitch $\Lambda=-0.5$ 

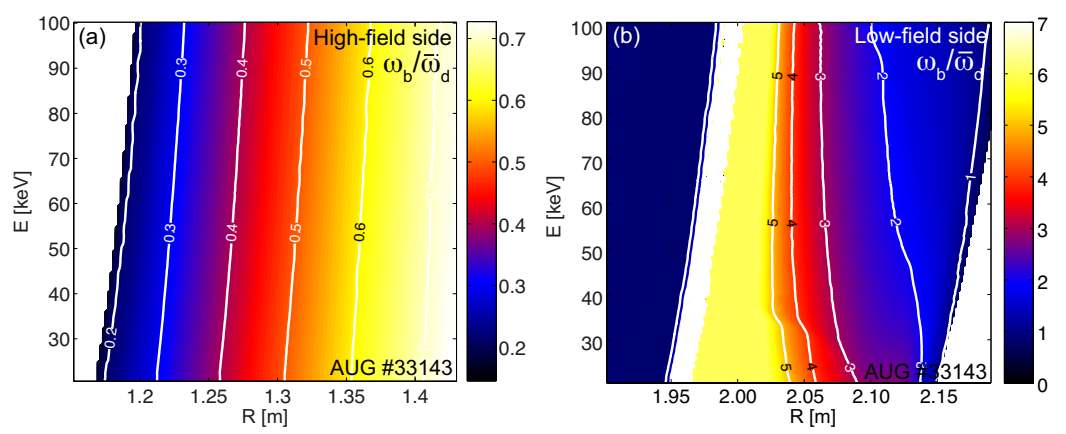

Figure 6. $A U G \# 33143$. Energetic particle resonances as a function of energy and plasma major radius of a NBI\#8 fast-ion distribution for (a) HFS using initial particle coordinates $\Lambda=-0.7, z=0 \mathrm{~m}$ and (b) LFS using initial particle coordinates $\Lambda=-0.5, z=0 \mathrm{~m}$.

been plotted for both high-field side (HFS) and low-field side (LFS), respectively. At the LFS, which is mainly populated by trapped orbits, the frequency ratio exhibits a small gradient in the region close to the scrape-off layer corresponding to deeply trapped ions. This gradient increases towards the plasma core as it approaches the trapped/passing boundary, resulting in a region with very high resonance density. In the high-field side region, where the population consists of passing orbits, the resonance gradient remains almost constant and the frequency ratio has a slight dependency on the particle initial energy.

A particle resonance will be considered linear if the trajectory frequency ratio meets the condition $n \bar{\omega}_{d}-p \omega_{b}=0$, where $p$ is the bounce harmonic. By overplotting the particle resonances in the $\left\langle\delta P_{\phi}\right\rangle$ figures of merit, a clear matching between the maxima and minima in $\left\langle\delta P_{\phi}\right\rangle$ and particle resonances is found. The particle resonances corresponding to a maximum variation of $\left\langle\delta P_{\phi}\right\rangle$ are $\omega_{b} / \bar{\omega}_{d}=2.25$ for $\Delta \varphi_{U L}=40^{\circ}, \omega_{b} / \bar{\omega}_{d}=2$ for $\Delta \varphi_{U L}=160^{\circ}$ and $\omega_{b} / \bar{\omega}_{d}=1.75$ for $\Delta \varphi_{U L}=260^{\circ}$. The matching between the maxima and minima of $\left\langle\delta P_{\phi}\right\rangle$ and the particle resonances indicates that, when the perturbation is applied, the ERTL induces fas-ion transport through a combination of linear and nonlinear resonances. As we approach the trapped/passing boundary, the distance between consecutive resonances is reduced, which might cause a significant $\left\langle\delta P_{\phi}\right\rangle$ due to a resonance overlap in this region. From the set of particle resonances shown in figure 6 , the ones responsible for the induced transport are located at the plasma edge, where the magnetic field is most affected by the 3D perturbation, as can be seen from the Poincaré maps in figures $2(\mathrm{~d})$ and (e) at $\rho=0.85-1.00$.

Most of the active resonances involved in the ERTL are nonlinear, which can be understood by considering a function describing varying field in the poloidal and toroidal direction in the form $f(r, \theta, \phi)=\sum_{n, m} e^{i n \phi-i m \theta} f_{m, n}(r)$ [22] in terms of particle coordinates $(r, \theta, \phi)$. The projection of the perturbation of the magnetic field along the 
trajectory of a trapped particle yields:

$$
f(r, \theta, \phi)=\sum_{n, m, p \in \mathbb{Z}} e^{i\left(n \bar{\omega}_{d}+p \omega_{b}-\omega_{0}\right) \tau} P_{n, m, p} \cdot f_{m, n}(r, \theta, \phi)
$$

where $\tau$ is a time variable that follows the particle location along the poloidal trajectory of period $2 \pi / \omega_{b}, \omega_{0}$ is the frequency of the perturbation and $P$ is a projection operator along the orbit of the trapped particle in an axisymmetric equilibrium [22]. Note that the aforementioned resonant condition can be trivially derived when the wave-particle phase becomes stationary, which leads to $n \bar{\omega}_{d}-p \omega_{b}=0$ for nearly stationary magnetic perturbations $\left(\omega_{0}=0\right)$.

When a linear resonant condition is not satisfied, we assume that the dominant impact of the perturbation is to modulate periodically the bounce-averaged particle displacement [23]. If we consider that the periodicity of the modulation is set by the magnetic perturbation and all terms are periodic functions which can be expressed using Fourier decomposition, we can write:

$$
f(r, \theta, \phi) \simeq \sum_{p, l \in \mathbb{Z}} e^{i\left(n \bar{\omega}_{d}-p \omega_{b}-\omega_{0}\right) \tau} e^{i l\left(n \bar{\omega}_{d}-p_{0} \omega_{b}-\omega_{0}\right) \tau} P_{n, m, p} \cdot f_{m, n}(r, \theta, \phi)
$$

for a fixed value of $n, m$, where $l$ is the nonlinear harmonic, $p=p_{0}+p^{\prime}$ is the nonlinear bounce harmonic and $p_{0}$ the primary bounce harmonic. From eq.4, the

resonant condition is given by $\frac{d}{d \tau}(\Omega \tau)=0$ with $\Omega=\left(n \bar{\omega}_{d}-p \omega_{b}\right)+l\left(n \bar{\omega}_{d}-p_{0} \omega_{b}\right)$ for stationary perturbations and can be expressed as:

$$
\frac{\omega_{b}}{\bar{\omega}_{d}}=\frac{n(1+l)}{p_{0}(1+l)+p^{\prime}}
$$

From expression 5 we can identify active resonances triggered by given values of $\Delta \varphi_{U L}$. In the analysed shot, $n=2$ is fixed due to the perturbation symmetry, $p^{\prime}= \pm 1$ and $p_{0}$ can take integer values reproducing most of the resonant structures in figures $5(\mathrm{a})-(\mathrm{c})$, where the amplitude and location of the activated resonance depends strongly on the frequency ratio.

\section{Characterisation of the ERTL}

Using the description of the ERTL as the main transport mechanism in the presence of a 3D perturbation generated by MP coils, a complete analysis of both LFS and HFS is presented in terms of $\left\langle\delta P_{\phi}\right\rangle$. The role of initial toroidal phase is illustrated in figure 7. Here, $\left\langle\delta P_{\phi}\right\rangle$ is calculated for different $\Delta \varphi_{U L}$ indicating the NBI\#8 injection. The structures in both configurations are dominated by the $n=2$ symmetry of the applied perturbation and the activation of multiple resonances at the trapped/passing boundary. Figure 7(a) indicates that, for this configuration of $\Delta \varphi_{U L}$, the NBI injection is the least favourable with respect to the perturbation, while figure 7(b) shows the opposite.

On the magnetic HFS, the variation of $\left\langle\delta P_{\phi}\right\rangle$ shows a different pattern since the fastion population in this area consists of passing particles (figures 8 (a)-(c)). However, 

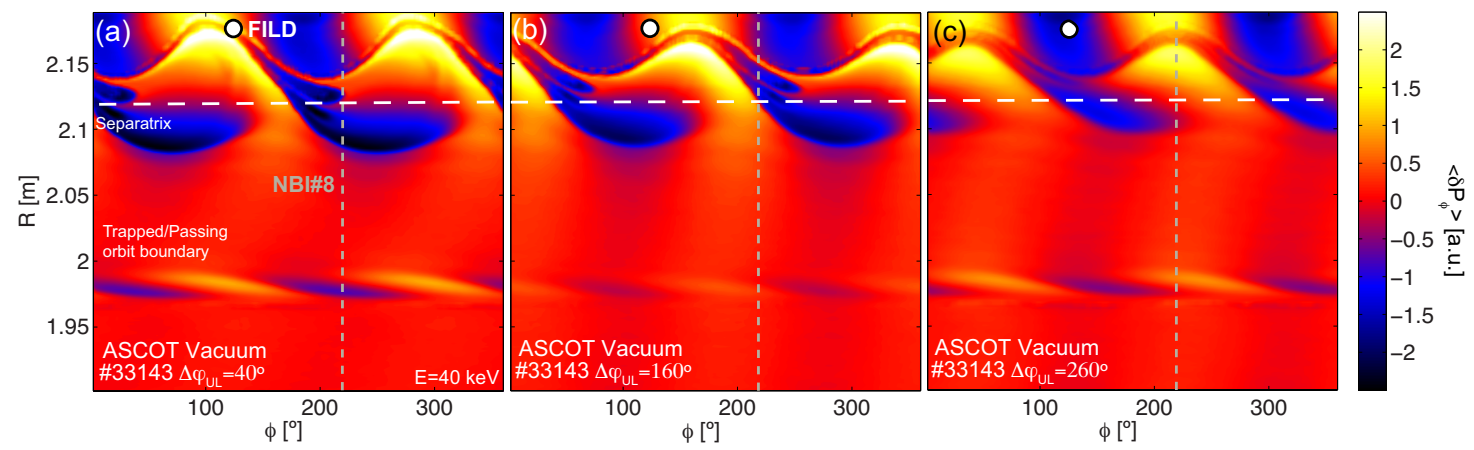

Figure 7. AUG\#33143. $\left\langle\delta P_{\phi}\right\rangle$ plot showing the LFS area using a particle distribution with initial coordinates $\Lambda=-0.5$ and $z=0 \mathrm{~m}$ as a function of plasma major radius and toroidal angle for: (a) $\Delta \varphi_{U L}=40^{\circ}$, (b) $\Delta \varphi_{U L}=$ $160^{\circ}$, (c) $\Delta \varphi_{U L}=260^{\circ}$. The dashed gray line indicates the beam injection of NBI\#8 and the location of the FILD detector is highlighted with a white circle.

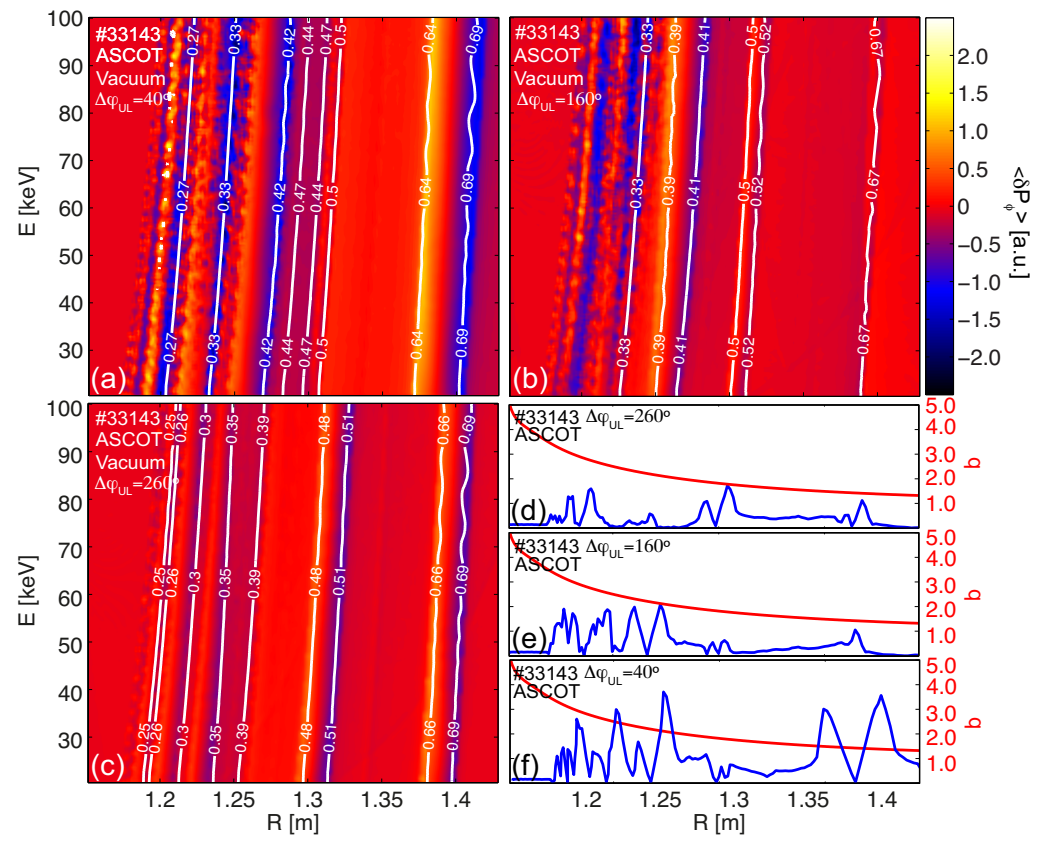

Figure 8. $A U G \# 33143 .\left\langle\delta P_{\phi}\right\rangle$ on the HFS area as a function of plasma major radius and energy for particle initial coordinates $\Lambda=-0.7$ and $z=0 \mathrm{~m}$ at: (a) $\Delta \varphi_{U L}=40^{\circ}$, (b) $\Delta \varphi_{U L}=160^{\circ}$, (c) $\Delta \varphi_{U L}=260^{\circ}$. (d)-(f) $\left|\left\langle\delta P_{\phi}\right\rangle\right|$ for different $\Delta \varphi_{U L}$ (blue) and q-profile (red) as a function of plasma major radius.

the variation of the poloidal mode spectra has a similar effect on the activated resonances, showing the maximum transport intensity at $\Delta \varphi_{U L}=40^{\circ}$ and minimum at $\Delta \varphi_{U L}=260^{\circ}$. The analysis of $\left\langle\delta P_{\phi}\right\rangle$ indicates that the transport is resonant in the inner plasma region, but becomes chaotic as it approaches the separatrix due to the perturbation of the magnetic field lines, which greatly affects passing particles.

For this case, the determination of the ratio $\omega_{b} / \bar{\omega}_{d}$ of the activated resonances was made by means of the identification of the $q=m / n$ values corresponding to the 
Characterisation of the ERTL Induced by 3D Fields in AUG through FO Simulations11
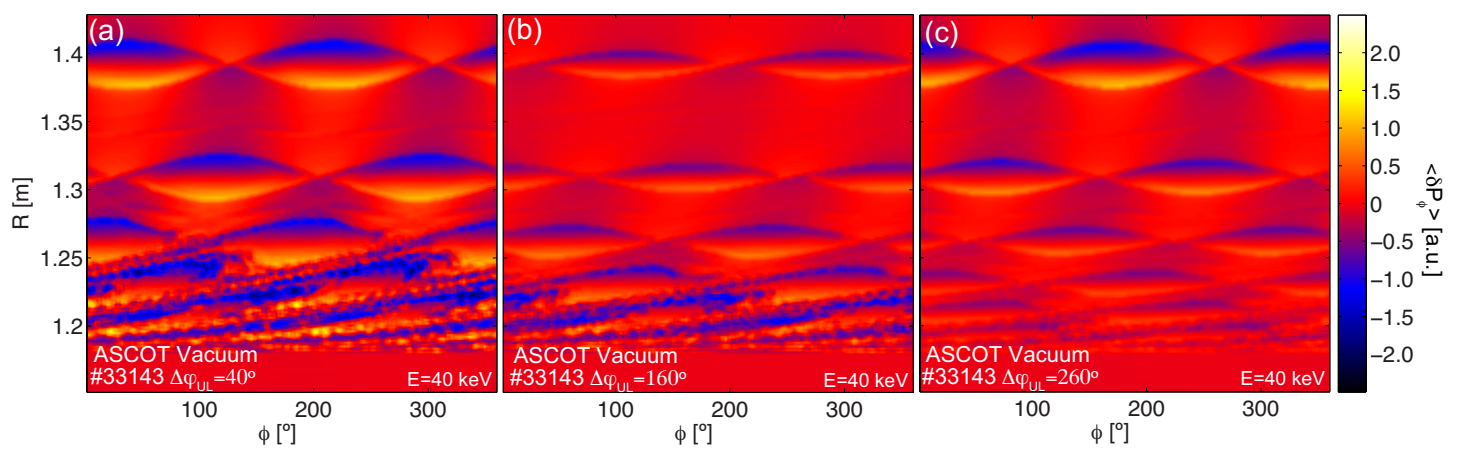

Figure 9. Variation of $P_{\phi}$ on the HFS area as a function of plasma major radius and toroidal angle for particle initial coordinates $\Lambda=-0.7$ and $z=0 \mathrm{~m}$ at: (a) $\Delta \varphi_{U L}=40^{\circ}$, (b) $\Delta \varphi_{U L}=160^{\circ}$, (c) $\Delta \varphi_{U L}=260^{\circ}$

peaks in the $\left\langle\delta P_{\phi}\right\rangle$ radial profile at a fixed value of the energy (figures $8(\mathrm{~d})$-(f)). The calculation of $\left\langle\delta P_{\phi}\right\rangle$ as a function of the plasma major radius and toroidal angle (figure $9)$ shows a resonant structure $(\mathrm{R}=1.25-1.45 \mathrm{~m})$ that is dominated by the $n=2$ toroidal mode number of the perturbation, which corresponds to the structures observed at the associated Poincaré plots (figure 2(e) and (f)). In addition to the $n=2$ structures, a lobe structure is visible at the region closer to the separatrix and is most intense when the resonant magnetic configuration is applied at $\Delta \varphi_{U L}=40^{\circ}$. Note that these structures showing the fast-ion transport at the high-field side are very similar to the divertor heat flux patterns observed in the AUG measurements with similar coil configurations [24]. A scan in the amplitude of the magnetic perturbation is used to analyse its effects on linear and nonlinear resonances (figures 10(a) and (b)). For amplitude levels routinely used for MP ELM suppression, both linear and nonlinear resonances are activated, but the resonant transport is mainly nonlinear (figure 10(a)). As the amplitude decreases, the intensity of nonlinear resonances $(l \neq 0)$ become weaker until they completely disappear and linear resonances are the dominant transport mechanism (figure 10(b)). This fading of the $\left\langle\delta P_{\phi}\right\rangle$ structures is summarized in figure10(d), where structures associated to linear resonances (marked with an orange circle) are the only structures that remain when the perturbation amplitude decreases.

The impact of Coulomb collisions on the ERTL is also assessed through realistic simulations including collisions between fast-ions and the bulk plasma where particle orbits have been traced using the same following time as figures 10 (a) and (b) to show the effect of collisions within the same time scale. The ASCOT collision operator includes pitch angle scattering [16] and uses a realistic value of the effective ion charge $\left(Z_{\text {eff }}=4.5\right)$ to determine the collision rates. In figures $10(\mathrm{c})$ and $(\mathrm{d})$ the impact of collisions on the ERTL leads to a widening of the resonances, but also to an overall decrease of the $\left\langle\delta P_{\phi}\right\rangle$ peak structure associated to the individual resonances.

The effect of the radial electric field on the ERTL is also studied. The radial electric field considered in figure11(a) was calculated for shot AUG\#33143 from the magnetic axis to the separatrix, showing the common behaviour observed in H-mode plasmas [25]. 
Characterisation of the ERTL Induced by 3D Fields in AUG through FO Simulations12

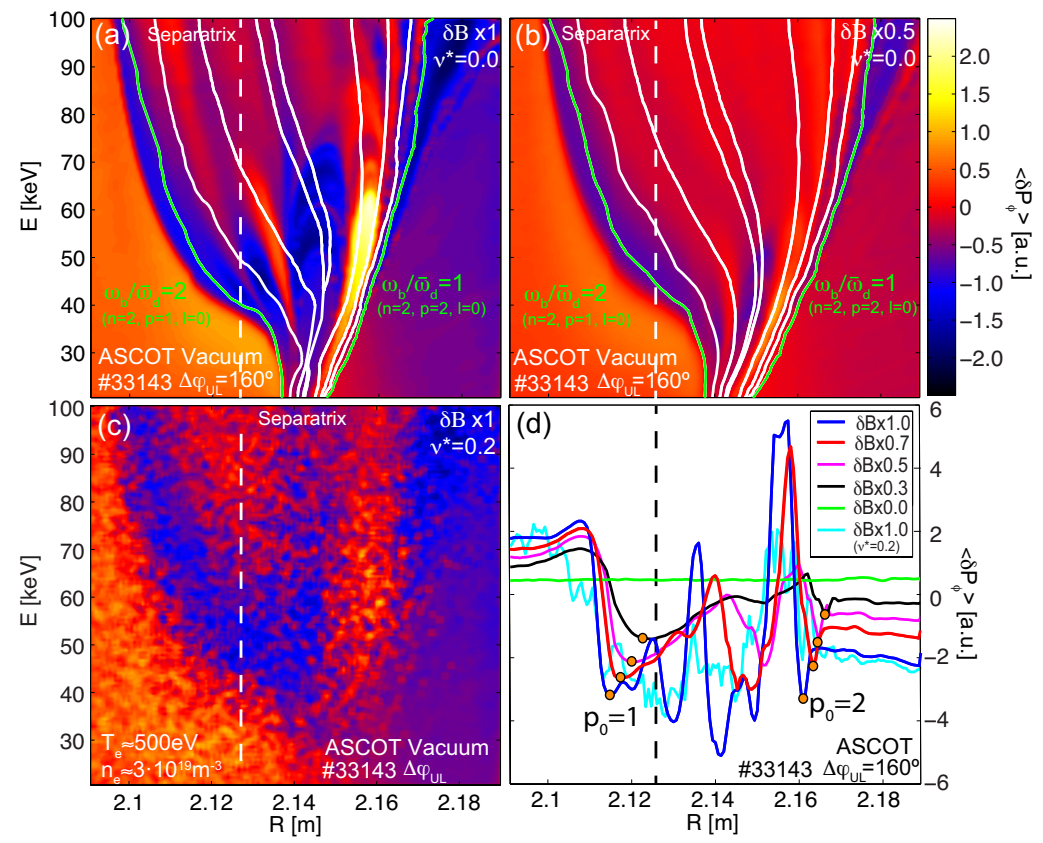

Figure 10. AUG\#33143. $\delta P_{\phi}$ calculated for $\Delta \varphi_{U L}=160^{\circ}$ coil configuration for a particle distribution initialised at $\Lambda=-0.5$ and $z=0 \mathrm{~m}$ using $3 D$ field amplitude of: (a) $\delta B \times 1$ (amplitude value from the measured coil currents), (b) $\delta B \times 0.5$ and (c) $\delta B \times 1$ including collisions. Contours indicate the $\omega_{b} / \bar{\omega}_{d}$ ratio for linear (green) and nonlinear (white) resonances. (d) $\left\langle\delta P_{\phi}\right\rangle$ profiles for $\Delta \varphi_{U L}=160^{\circ}$ configuration at energy $E=50 \mathrm{keV}$ using different $\delta B$ amplitudes where linear resonances are indicated by orange circles.

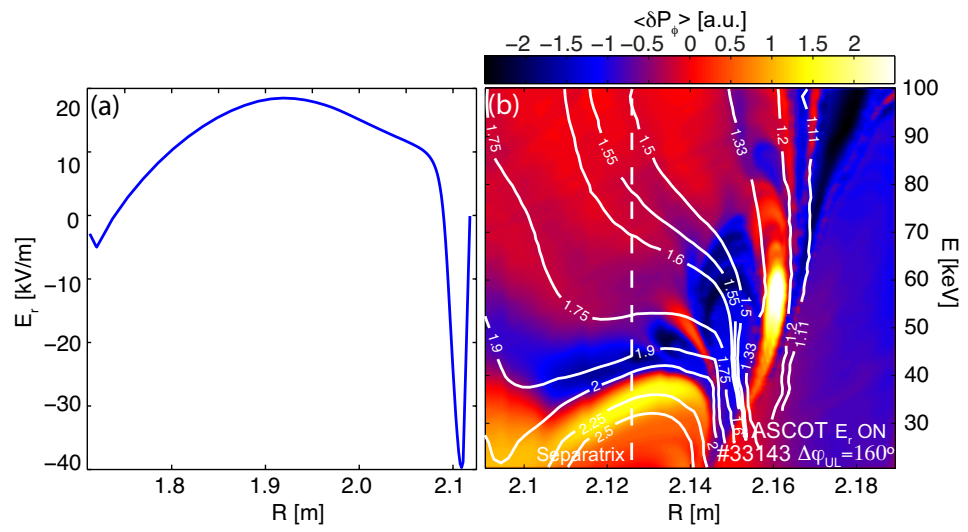

Figure 11. AUG\#33143. (a) Radial electric field profile derived from AUG experimental measurements. (b) $\left\langle\delta P_{\phi}\right\rangle$ calculated for $\Delta \varphi_{U L}=160^{\circ}$ coil configuration including the radial electric field for particle initial coordinates $\Lambda=-0.5$ and $z=0 \mathrm{~m}$. White contours indicate the particle frequency ratio $\omega_{b} / \bar{\omega}_{d}$. 
As can be observed from the comparison between figures 11(b) and 10(a), the impact of the radial electric field is to distort the resonant structures at the location of the $E_{r}$ well, but it can also alter a small region outside the separatrix. This effect is related to the topology of trapped orbits (figure $2(\mathrm{c})$ ), where ions born outside the separatrix can also explore inner regions of the plasma on the inner banana orbit leg. The orbital frequency ratio $\omega_{b} / \bar{\omega}_{d}$ was calculated including the $E_{r}$, showing that the distortion of the $\left\langle\delta P_{\phi}\right\rangle$ structures matches the new frequency ratio. Considering this, we can assume that eq. 5 is still valid in the presence of $E_{r}$.

Although the structures are altered, the maximum variation of $\left\langle\delta P_{\phi}\right\rangle$ is not changed. Also, due to the distortion of the resonances, the transport associated to $\left\langle\delta P_{\phi}\right\rangle$ at high energies is eliminated, which might lead to a better ion confinement for this coil configuration.

\section{Summary}

This work introduces an Edge Resonant Transport Layer responsible for the fast-ion losses in the presence of externally applied 3D fields in a tokamak. This transport mechanism is shown to have a dependency on the energetic particle topology, the spatial spectrum and amplitude of the perturbation, plasma collisions and radial electric field $\left(E_{r}\right)$. The dominant resonant transport is mainly linear for small amplitude perturbations, but it becomes nonlinear as the amplitude increases to levels used routinely for MP ELM suppression. When Coulomb collisions are included, the waveparticle resonant phase is lost more rapidly than in the collisionless case and, hence, the transport decreases. Also, when a radial electric field is considered, a distortion of the resonant patterns is observed near the $E_{r}$ well, but the transport remains close to the separatrix with a similar maximum variation of $\left\langle\delta P_{\phi}\right\rangle$. The results discussed in this work were used to understand the nature of the fast-ion transport mechanism involved in the analysed experiment, but these modelling tools can also be utilized to predict the energetic particle confinement in current and future devices like ITER. Based on this, the analysis of $\left\langle\delta P_{\phi}\right\rangle$ can be applied to optimize future operational scenarios by modifying the ERTL, which opens the possibility of fast-ion control through the application of 3D perturbations.

The role that the ERTL introduced here may play on the observed mitigation and suppression of ELMs in present devices will be studied in detail in a follow-up paper including its dependence on $q_{95}$, collisionality and $E_{r}$. This future work might allow us to identify a 3D field configuration that maximizes the ELM mitigation/suppression and minimizes the loss of superthermal ions.

\section{Acknowledgments}

This work has been carried out within the framework of the EUROfusion Consortium and has received funding from the Euratom research and training program 2014-2018 
under grant agreement number 633053. The views and opinions expressed herein do not necessarily reflect those of the European Commission. The simulations were partly performed on the MARCONI supercomputer (CINECA) under project reference FUA32_ASCOT-US. D. Z. acknowledges support from the A*MIDEX project (no. ANR11-IDEX-0001-02) funded by the Investissements d'Avenir French Government program, managed by the French National Research Agency (ANR).

\section{References}

[1] T C Hender et al. Effect of resonant magnetic perturbations on COMPASS-C tokamak discharges. Nucl. Fusion, 32:2091, 1992.

[2] T E Evans et al. Suppression of Large Edge-Localized Modes in High-Confinement DIII-D Plasmas with a Stochastic Magnetic Boundary. Phys. Rev. Lett, 92(23):235003, 2004.

[3] Y Liang et al. Active control of type-I edge-localized modes with $\mathrm{n}=1$ perturbation fields in the JET tokamak. Phys. Rev. Lett, 98(26):265004, 2007.

[4] W Suttrop et al. First Observation of Edge Localized Modes Mitigation with Resonant and Nonresonant Magnetic Perturbations in ASDEX Upgrade. Phys. Rev. Lett, 106:225004, 2011.

[5] Jong-Kyu Park. Control of Asymmetric Magnetic Perturbations in Tokamaks. Phys. Rev. Lett, 99(10):195003, 2007.

[6] M Willensdorfer et al. Field-Line Localized Destabilization of Ballooning Modes in ThreeDimensional Tokamaks. Phys. Rev. Lett, 119(8):085002, 2017.

[7] C Paz-Soldan et al. Observation of a multimode plasma response and its relationship to density pumpout and edge-localized mode suppression. Phys. Rev. Lett, 114(10):105001, 2015.

[8] R Nazikian et al. Pedestal Bifurcation and Resonant Field Penetration at the Threshold of EdgeLocalized Mode Suppression in the DIII-D Tokamak. Phys. Rev. Lett, 114(5):105002, 2015.

[9] T Kurki-Suonio et al. Protecting ITER walls: fast ion power loads in 3D magnetic field. Plasma Phys. Control. Fusion, 59:014013, 2017.

[10] K Shinohara et al. Effects of complex symmetry-breakings on alpha particle power loads on first wall structures and equilibrium in ITER. Nucl. Fusion, 51:63028, 2011.

[11] K Shinohara et al. Effects of rippled fields due to ferritic inserts and ELM mitigation coils on energetic ion losses in a 15 MA inductive scenario in ITER. Nucl. Fusion, 52:094008, 2012.

[12] M.A. Van Zeeland et al. Fast ion transport during applied 3D magnetic perturbations on DIII-D. Nucl. Fusion, 55:073028, 2015.

[13] G J Kramer et al. Fast-ion effects during test blanket module simulation experiments in DIII-D Simulation of localized fast-ion heat loads in test blanket module simulation experiments on DIII-D A description of the full-particle-orbit- following SPIRAL code for simulating. Nucl. Fusion, 51:103029, 2011.

[14] M Garcia-Munoz et al. Fast-ion transport induced by Alfvén eigenmodes in the ASDEX Upgrade tokamak. Nucl. Fusion, 51:103013, 2011.

[15] M. Garcia-Munoz et al. Fast-ion losses induced by ELMs and externally applied magnetic perturbations in the ASDEX Upgrade tokamak. Plasma Phys. Control. Fusion, 55:124014, 2013.

[16] E Hirvijoki et al. ASCOT: Solving the kinetic equation of minority particle species in tokamak plasmas. Comput. Phys. Commun, 185:1310, 2014.

[17] Yueqiang Liu, M S Chu, W F Guo, and F Villone. Resistive Wall Mode Control Code Maturity. Plasma Phys. Control. Fusion, 52:104002, 2010.

[18] M Willensdorfer et al. Three dimensional boundary displacement due to stable ideal kink modes excited by external $\mathrm{n}=2$ magnetic perturbations. Nucl. Fusion, 57:116047, 2017.

[19] M Garcia-Munoz, H.-U Fahrbach, and H Zohm. Scintillator based detector for fast-ion losses 
induced by magnetohydrodynamic instabilities in the ASDEX upgrade tokamak. Rev. Sci. Instrum., 80(81):053503, 2009.

[20] M J Lanctot et al. Validation of the linear ideal magnetohydrodynamic model of three-dimensional tokamak equilibria. Nucl. Fusion, 17:030701, 2010.

[21] W Schneider et al. Three dimensional boundary displacement due to stable ideal kink modes excited by external $\mathrm{n}=2$ magnetic perturbations. Fusion Eng. Des., 48:127?134, 2000.

[22] F Zonca, L Chen, S Briguglio, G Fogaccia, G Vlad, and X Wang. Nonlinear dynamics of phase space zonal structures and energetic particle physics in fusion plasmas. New J. Phys., 17(17):013052, 2015.

[23] L. Chen and F. Zonca. Physics of Alfven waves and energetic particles in burning plasmas. Rev. Mod. Phys., 88:015008, 2016.

[24] M. Faitsch, B. Sieglin, T. Eich, A. Herrmann, and W. Suttrop. 2D heat flux in ASDEX Upgrade L-Mode with magnetic perturbation. Nuclear Materials and Energy, 12:225004, 2017.

[25] E. Viezzer et al. High-accuracy characterization of the edge radial electric field at ASDEX Upgrade. Nucl. Fusion, 53(5):053005, 2013. 